\title{
La sistematización como metodología de la educación popular y su utilización para la mejora de la formación pedagógica del profesor universitario
}

\section{The systematizing like methodology of the popular education and their use for the improvement of the university professor's pedagogic formation}

\begin{abstract}
RESUMEN
El profesor universitario ejerce una segunda profesión, ya que es graduado en diferentes ramas del saber, pero realiza la función docente; esto fundamenta la necesidad de su formación para esa actuación profesional. El propósito del trabajo consiste en una revisión bibliográfica y documental para resaltar el papel de la sistematización de la formación pedagógica que se realiza en la universidad y develar la contribución de su utilización para el mejoramiento de las diferentes alternativas que se utilizan en la educación superior. La sistematización como metodología de la Educación Popular posibilita la mejora de la formación pedagógica del docente universitario. Estudios de la temática, como los realizados por Paulo Freire, corresponden a las dimensiones de la formación pedagógica del docente universitario, así como resultado de la experiencia de las autoras del trabajo se corrobora la necesidad de buscar alternativas formativas que involucren activamente en su desarrollo a los docentes que la requieren. La investigación realizada posibilita perfeccionar y ordenar un sistema que integra lo instructivo y lo educativo en la formación del claustro, lo que contribuye a la formación docente para un mejor desempeño.
\end{abstract}

Palabras clave: formación pedagógica, sistematización, profesor universitario

\footnotetext{
${ }^{1}$ Universidad de Cienfuegos "Carlos Rafael Rodríguez", Cienfuegos, Cuba.

2 Universidad de Ciencias Médicas, Cienfuegos, Cuba.

3 Universidad de Cienfuegos "Carlos Rafael Rodríguez", Cienfuegos, Cuba.
} 


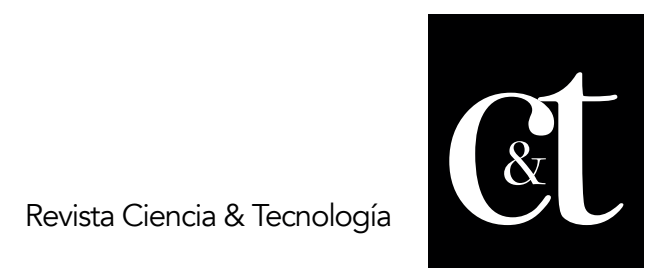

No. 27, 31 de julio de 2020

ISSN impreso: 1390 - 6321

ISSN online: 2661 - 6734

\begin{abstract}
The university professor exercises a second profession, since it is graduated in different branches of the knowledge, but he carries out the educational function, what bases the necessity of his formation for that professional performance. The purpose of the work consists of a bibliographic and documentary review to stand out the paper of the systematizing of the pedagogic formation that is carried out in the university and reveal the contribution of its use for the alternative improvement of the different ones that you/they are used in the superior education. The systematizing like methodology of the Popular Education facilitates the improvement of the educational university student's pedagogic formation. According to results of a questionnaire applied at 604 educational composed by 25 articles that correspond to the dimensions of the educational university student's pedagogic formation, as well as result of the experience of the authors of the work is corroborated the necessity to look for alternative formative that involve actively in its development to the educational ones that require it. The current investigation facilitates to perfect and to order a system that integrates the instructive thing and the educational thing in the formation of the cloister, what contributes to the educational formation for a better acting.
\end{abstract}

Keywords: pedagogic formation, systematizing, university professor

\title{
Introducción
}

Las universidades son las encargadas no solo de formar profesionales, sino además de garantizar la formación posgraduada de profesionales egresados de la educación superior, que requieren formación en cuarto nivel de enseñanza, la que se dinamiza significativamente en las últimas tres décadas, por los cambios en las ramas del saber y del desarrollo acelerado de la ciencia, las tecnologías y las comunicaciones.

Existen propuestas en las universidades para formar a sus docentes universitarios, entre las que se destacan cursos, entrenamientos y diplomados; y conferencias, seminarios y talleres temáticos. En ellos se debaten temas con los retos actuales de las más disímiles profesiones, para actualizar conocimientos en el cuarto nivel de enseñanza; todas estas variantes señaladas son formas de superación profesional postgraduada, que complementa la formación académica (Baute e Iglesias, 2010).

Por su naturaleza la sistematización se reconoce como una tendencia progresista. Parte del análisis crítico de las realidades socioeducativas ve la crítica como ejercicio en el que se articulan lo tradicional y lo renovador a partir de la participación social.

Como teoría tiene un enfoque epistemológico de amplia aplicación en las sociedades interesadas en potenciar la independencia, la autorrealización y la transformación del hombre, quien debe superar la opresión de las formas de pensar estandarizadas. Se orienta a estimular el desarrollo individual y colectivo de sus individuos y a su vez, se centra esencialmente en el desarrollo integral de la personalidad.

La sistematización plantea desarrollar capacidades para que los sujetos procesen información, manejen estímulos del medio, practiquen el razonamiento cognitivo, y puedan aprender y valorar críticamente experiencias pedagógicas. Exige niveles de implicación al usar como referente criterios de los participantes.

Se comparte los criterios que conciben la sistematización como una fuente de conocimientos muy vinculada a la investigación y al saber pensar, inferir, analizar los fenómenos que la experiencia propone transformar y adecuar a las necesidades 


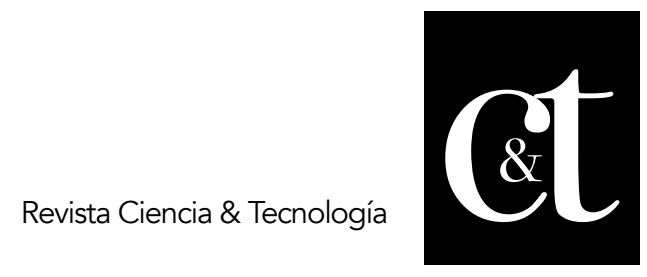

No. 27, 31 de julio de 2020

ISSN impreso: 1390 - 6321

ISSN online: 2661 - 6734

individuales y colectivas del conocimiento, las habilidades, valores y modos de actuación, por lo que la sistematización contribuye a la mejora de la formación pedagógica permanente del profesor universitario. El objetivo del trabajo radica en abordar mediante la metodología de la sistematización la formación pedagógica que se realiza en la universidad y develar la importancia de la utilización para el mejoramiento de las diferentes alternativas que se utilizan en la educación superior.

\section{Desarrollo}

La teorización acerca de la sistematización está asociada a concepciones de transformación de la práctica social y como posición crítica a la pedagogía bancaria o tradicional. Su origen se identifica con el desarrollo de experiencias comunitarias y pedagógicas en América Latina en la década del 70 del siglo XX, interesadas en promover el proceso educativo a partir de explicaciones verdaderas y contemplar la historicidad de las IES, comunidades o procesos sociales orientados a la búsqueda de la emancipación mediante la participación activa de los sujetos en la transformación.

Por su naturaleza se reconoce como una tendencia progresista. Parte del análisis crítico de las realidades socioeducativas, considera la crítica como ejercicio trasformador que articula tradición y lo renovación a partir de la participación social.

Como teoría tiene un enfoque epistemológico de amplia aplicación en las sociedades interesadas en potenciar la independencia, la autorrealización y la transformación. El humano debe superar la opresión de estandarizaciones. Se orienta a estimular el desarrollo individual y colectivo de sus individuos y a su vez, se centra esencialmente en el desarrollo integral de la personalidad. Esta orientación destaca su intención de superar enfoques dualistas de separar el individuo y sociedad. Considera el carácter activo de los procesos psíquicos, la actividad (interna y externa) y la comunicación.

El aprendizaje es actividad de carácter social, es activo y orientador; de ahí su objetivo: develar las capacidades, en niveles reales y potenciales, en proceso de despliegue, redescubrimiento y construcción del conocimiento, usando los escenarios de colaboración que las condiciones permitan en las prácticas a sistematizar. Responde a una concepción desarrolladora del aprendizaje y tiene raíces en el pensamiento pedagógico de Freire (1981-1992), quien advierte la posibilidad de ser considerada la sistematización como un método de todo proyecto socioeducativo en el que se proponga el desarrollo humano y la transformación comunitaria; en ella hay un proceso creador de nuevos conocimientos, habilidades, valores y modos de actuación.

Diferentes estudiosos de la obra de Paulo Freire desarrollan concepciones sobre la sistematización y sus aportes al desarrollo del conocimiento por medio del análisis reflexivo y crítico de las prácticas, donde los actores tienen un papel importante en el desarrollo de la teoría desde la práctica. Ellos sustentan sus teorías en el estudio de la teoría que sustenta la Educación Popular desarrollada por Paulo Freire.

Los investigadores y promotores de esta teoría insisten en que la sistematización puede definirse como un ejercicio de reflexión sobre la experiencia, que permite comprender, ordenar los procesos, los resultados de un proyecto, buscando en tal dinámica las dimensiones que pueden explicar el curso que asumió el trabajo realizado. Puede identificarse también como un método permanente, acumulativo, de creación de conocimientos a partir de la experiencia de intervención en una 


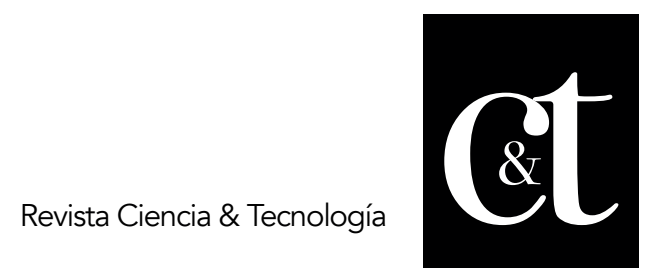

No. 27, 31 de julio de 2020

ISSN impreso: 1390 - 6321

ISSN online: 2661 - 6734

realidad social, como un primer nivel de teorización sobre la práctica (Jara Holliday, 2008). Expresa, por tanto, una articulación entre teoría y práctica que apunta a mejorar la práctica y la intervención desde lo que ella misma enseña.

Como Freire demuestra, desde la práctica se aspira a enriquecer, confrontar y modificar, el conocimiento teórico actualmente existente, contribuyendo a convertirlo en una herramienta realmente útil para entender y transformar la realidad.

En sus estudios Freire (2015) aprecia en la sistematización una modalidad participativa de producción de conocimientos sobre la práctica educativa que, a partir de su reconstrucción, ordenamiento descriptivo e interpretación crítica, busca cualificarla y comunicarla, y la considera una experiencia educativa en sí misma para los que participan en ella, ya que produce conocimientos y socializa el conocimiento generado, resaltando así, su condición de carácter participativo, el cual se relaciona con la posibilidad para buscar, penetrar en la dinámica de las experiencias, identificando las relaciones entre ellos, recorriendo sus diferentes etapas, localizando sus contradicciones, tensiones, marchas y contramarchas, llegando así a entender dichos procesos desde su propia lógica, extrayendo de allí enseñanzas que puedan aportar al enriquecimiento tanto de la práctica como de la teoría.

En tal sentido, las autoras de esta reflexión, aprecian en la sistematización el desarrollo de las capacidades para que los sujetos procesen información, manejen estímulos del ambiente, practiquen razonamiento cognitivo, y desarrollen capacidad para aprender, asimilar y valorar críticamente experiencias pedagógicas. Exige un nivel de implicación al utilizar como referente los propios criterios de los participantes. Se comparte los criterios que conciben la sistematización como una fuente de conocimientos muy vinculada a la investigación y al saber pensar, inferir, analizar los fenómenos que la experiencia propone transformar y adecuar a las necesidades individuales y colectivas del conocimiento, las habilidades, valores y modos de actuación, por lo que la sistematización contribuye a la mejora de la formación pedagógica del profesor universitario.

A partir del análisis de la definición de sistematización las autoras consideran que la sistematización constituye proceso de transformación de conocimientos, habilidades, valores y modos de actuación del profesor universitario mediante la investigación que realiza el docente de su propia práctica orientada hacia el cambio educativo.

Se considera que las características de la sistematización de la formación pedagógica son varias; en primer lugar se construye desde y para la práctica, requiriendo la presencia de registros con el propósito de reconstruir saberes, con el fin de mejorar la práctica mediante su trasformación, también procura comprenderla; requiere de la participación de los que participan en la mejora de sus propias prácticas; exige una actuación grupal por la que los implicados (profesores, directivos -académicos, profesores que matriculan en las ofertas formativas y coordinador de la experiencia a sistematizar), colaboran coordinadamente en el proceso de teorización; implica el análisis crítico de las situaciones para transformar la realidad desde la elaboración de nuevos conocimientos teóricos y prácticos (el resultado de la sistematización).

La sistematización declarada en el párrafo anterior por las autoras, puede ser realizada en el marco de modalidades de formación pedagógica que se le ofrecen al profesor universitario, lo que contribuye a estrechar la distancia hoy existente entre las prácticas docentes del profesor y los contenidos teóricos que proponen las ofertas 


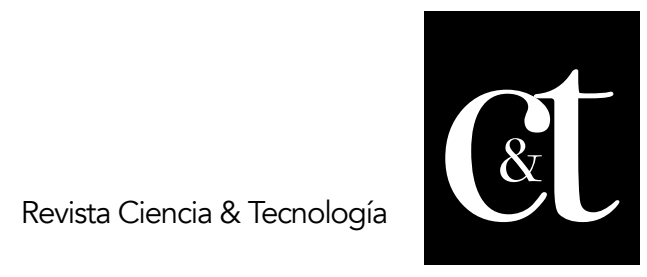

No. 27, 31 de julio de 2020

ISSN impreso: 1390 - 6321

ISSN online: 2661 - 6734

de formación. Tal combinación estimula el perfeccionamiento de la formación pedagógica y a su vez la realización de investigaciones en el marco de la formación pedagógica que desarrolle en los docentes la actuación requerida para responder desde la teoría a la práctica, generando nuevos conocimientos transformadores.

Como demostró Freire, la sistematización de experiencias posibilita por tanto, desarrollar una práctica profesional dinámica, única, permanente e irrepetible del proceso enseñanza-aprendizaje, en nuestro caso, en la formación docente, contribuyendo al perfeccionamiento de la formación por el requerida para transformar el proceso de enseñanza-aprendizaje en el que participa el profesor de manera protagónica derivado de las teorizaciones constantes de los problemas y desafíos que encuentra en su profesión como docente en la Educación Superior.

La transferencia de este método de investigación a los procesos de formación del docente es aún muy nueva. Existen autores que la asocian a procesos de investigación educativa que desarrollan los docentes. En este sentido aluden a la necesidad de la sistematización profesional como proceso permanente, que incrementa la autopreparación docente, y que tiene como propósito la autogestión del conocimiento y la creación de condiciones de trabajo que permiten aportar alternativas de solución y tareas profesionales, lo que posibilita el autodesarrollo personal y profesional mediante el cumplimiento de sus funciones lo que exige una interpretación y valoración crítica de varias experiencias, según García y Addine (2006) y Fuentealba (2014).

En tal sentido, la sistematización de manera coincidente legitima su contribución a la práctica mediante el estudio de experiencias por parte de los docentes que de manera participativa valoran sus prácticas y tratan de transformarlas por medio del análisis reflexivo en talleres, no siempre propiciando desarrollo de conocimientos teóricos desde la práctica como resultado de una investigación con un enfoque pedagógico.

Dar cumplimiento a las exigencias de la sistematización de la enseñanza demanda que los profesores no solo se apropien de un sistema de conocimientos, sino también lo desarrollen mediante pensamiento integrado por distintas operaciones lógicas: análisis-síntesis, generalización, abstracción, inducción y deducción, que conduzcan al desarrollo teórico y práctico de las Ciencias Pedagógicas y ofrezcan solución a los problemas de su práctica docente y se perfeccione a su vez la formación permanente del docente; así lo han entendido también Mejía (2014) y Lucio-Villegas (2015).

Las autoras coinciden con los que valoran la sistematización como un proceso permanente, acumulativo, de creación de conocimientos a partir de la práctica, que requiere formas de formación que propicien a los docentes las herramientas requeridas para que se apropien del método de sistematización y lo incorporen a su quehacer como alternativa de enriquecimiento constante de la práctica docente, con el fin de solucionar problemas prácticos, produce conocimiento y lo socializa, constituyendo un requisito para desarrollar teoría y práctica docente en la actualidad.

Se considera que organizar la sistematización de la formación pedagógica permite definir con claridad los componentes que posibilitan su sistematización, que luego se derivan en acciones, lo que exige precisar con claridad: el objetivo, el objeto y el eje temático de la sistematización, así como determinar el formato a utilizar para el registro de la información que se pretende sistematizar, los recursos, tiempo, actores, entre otros elementos donde no pueden faltar preguntas que den respuesta 


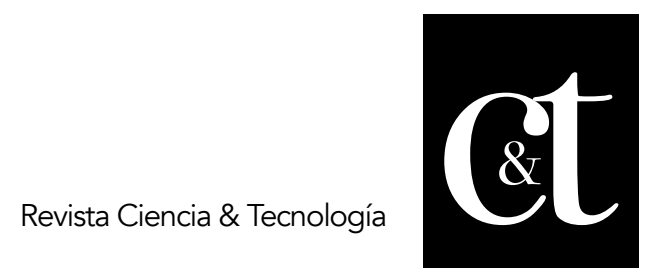

No. 27, 31 de julio de 2020

ISSN impreso: 1390 - 6321

ISSN online: 2661 - 6734

a los elementos planteados en cada una de las etapas progresivas a seguir durante la investigación.

El rigor del método utilizado no exime la flexibilidad en orientar las discusiones, en la prioridad de ejes de sistematización que se definan, los que se corresponderán con el nivel de desarrollo de los implicados, intereses de perfeccionamiento y, sobre todo, con la situación que presenta el proceso en cuestión (vías de la formación pedagógica que se decidan sistematizar). Los resultados de la reflexión que provoca la sistematización se asumen como plataforma para teorizar, elaborar materiales orientadores y asimilar estudios de investigación como alternativas de solución o acciones que aseguren continuidad del proceso de formación pedagógica.

Desde esta óptica la sistematización como demostró Freire en sus prácticas comunitarias, genera nuevos conocimientos acerca de cómo marcha el proceso de transformación de la práctica mediante la participación activa de todos los participantes en su transformación, lo que constituía su objetivo fundamental. Para el caso de la actual propuesta demuestra cómo se generan nuevos conocimientos en los docentes que participan en la formación pedagógica para transformar el proceso de enseñanza -aprendizaje en las distintas modalidades de la formación pedagógica, replanteándose cada uno de los elementos didácticos que presenten dificultades o precisen contextualización. Incluye la activa presencia de participantes y profesores, apoyándose en recursos y técnicas que legitimen la objetividad, y pertenencia de los análisis realizados por los profesores que cursan las ofertas formativas y sus profesores, convirtiéndolos en postulados teórico-prácticos enriquecedores.

Se considera que, para lograr éxito en la sistematización, como demostró Freire, tiene prioridad definir el papel y las funciones de cada participante. Teniendo en cuenta los actores (profesores, profesores que cursan las ofertas, directivosacadémicos y coordinador), fueron secuenciados los ejes de sistematización y las técnicas e instrumentos utilizados para la recopilación y registro de toda la información, como requisito para involucrarlos en todos los momentos desde el registro, hasta la transformación de la práctica mediante las investigaciones e innovaciones.

El profesor/alumno: sus vivencias, sus aprendizajes, su visión, sus expectativas sobre la oferta de formación pedagógica a la que se incorpora y las necesidades de formación pedagógica escalonada partiendo de su práctica para teorizarla y volver a ella para transformarla. Las modalidades formativas no siempre tienen la premisa de la práctica, existiendo evidencias documentales de algunas ofertas ofrecidas a los docentes con dominio de contenido teórico y cierto alejamiento del contexto donde se desarrolla la práctica docente.

El profesor (quien imparte formación pedagógica): su propia práctica, la participación de los 'profesores que participan, los resultados, las contradicciones que se generan entre las propuestas del profesor para desarrollar el curso y las necesidades de aprendizajes de los que participan para mediante la teorización transformar su práctica docente. Deberán buscarse alternativas para su transformación y la utilización de formas colaborativas de enriquecer y/o transformarlas y desarrollar la teoría que dé respuesta a los conocimientos, habilidades, valores y modos de actuación a los que tributa la modalidad formativa en cuestión. 


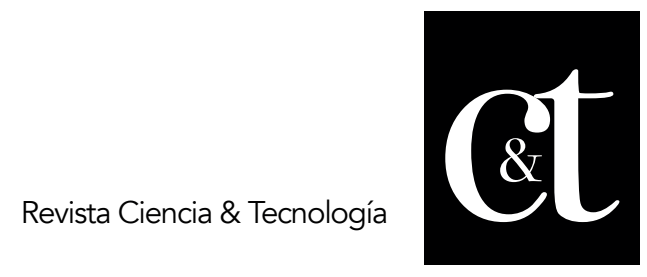

No. 27, 31 de julio de 2020

ISSN impreso: 1390 - 6321

ISSN online: 2661 - 6734

Los directivos- académicos (son los que dirigen al profesor y aporta valoraciones importantes sobre las necesidades de formación): valoraciones que ofrece el directivo- académico sobre la actuación docente, la relación entre lo que se enseña y las exigencias de la práctica, los aprendizajes que actualiza, las vías que puede utilizar para perfeccionar la superación de los profesores, así como, los aprendizajes relacionados con el proceso de enseñanza-aprendizaje en el postgrado desarrollador de la autogestión de los propios participan de sus necesidades de conocimientos, habilidades y modos de actuación para transformar su práctica mediante la sistematización, son elementos claves que retroalimentan y transforman la actuación del docente, luego de culminada la formación en que participa. Esta interrelación directivo académico, profesor del curso y profesor-alumno potencia el compromiso de todos por la calidad de la formación pedagógica y su seguimiento posterior al observar los cambios en los modos de actuación del docente (González, 1999).

Se comparte el criterio de que la reconstrucción de toda la experiencia es momento de aprendizaje colaborativo, puesto que es un primer nivel de socialización de las experiencias que constituyen antecedentes empíricos de la transformación a realizar mediante la teorización, y conducen al desarrollo y retroalimentación necesaria para nuevos procesos de sistematización.

En síntesis, la sistematización de la formación pedagógica propuesta por las autoras permitió profundizar en el pensamiento freiriano y su teoría sobre la Educación Popular, su vigencia y transferibilidad a diferentes objetos de investigación social.

La sistematización de la formación pedagógica del docente contribuye al fortalecimiento de la identidad profesional del educador, confirma la ética que debe legitimarse; los modos de actuación y valores que se correspondan con las exigencias sociales para el momento histórico-social en que tienen lugar, lo que requiere su contextualización a diferentes realidades educativas y deben quedar fortalecidas durante la propia formación pedagógica, pues esta se traza objetivos instructivos y educativos fortalecedores de los modos de actuación del profesor universitario. El pensamiento de Freire permite constatar su importancia y actualidad, así como el rol de su metodología para transformar la práctica social.

Propició, a todos los participantes en el estudio, enseñanzas significativas como mayor participación de directivos académicos y profesores en la sistematización que de manera conjunta y crítica valoran la superación pedagógica actual, sus fortalezas y debilidades con el fin de transformarlas de manera conjunta y participativa, lo que conciencia la necesidad de formación pedagógica, personalizada, integrada, contextualizada, con mayor flexibilización en función del diagnóstico de necesidades y su vínculo con las demandas de las ciencias pedagógicas contemporáneas con respuesta al modelo pedagógico dominante, al liderazgo del profesor en su práctica docente y al perfeccionamiento de su formación (Freire, 2019).

Se confirma la necesidad de revisar contenidos, habilidades, valores y modos de actuación a los que responde cada modalidad formativa sistematizada, por la necesidad de incorporar nuevas necesidades de superación detectadas por los profesores que imparten las ofertas de superación pedagógica y otras necesidades de formación identificadas por los participantes en la formación pedagógica en las alternativas de sistematización proyectadas. Se valora de manera crítica el objetivo general, los contenidos, habilidades, métodos, medios y formas evaluativas de los diferentes cursos de formación pedagógica para evitar la repetición de contenidos y 


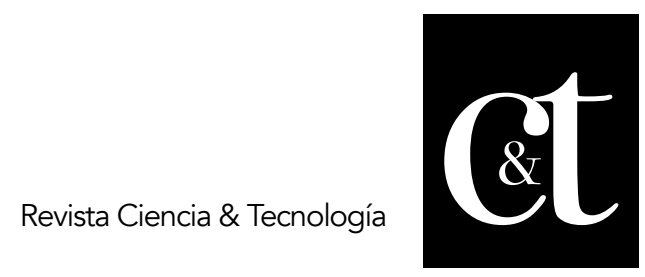

No. 27, 31 de julio de 2020

ISSN impreso: 1390 - 6321

ISSN online: 2661 - 6734

propiciar el paso de conocimientos menos a más complejos en la medida que se transita por las formas de superación de manera escalonada y progresiva.

Se introducen y actualizan contenidos pedagógicos, derivándose de ellos nuevos conocimientos didácticos a la luz de las tecnologías de la información y las comunicaciones actuales y su incidencia en la formación de profesionales y así como otros cursos que sean de interés de los propios profesores insertados en la sistematización, fortaleciéndose el nivel del diplomado como oferta de formación pedagógica que contribuye al perfeccionamiento de la práctica docente del profesor universitario e incentiva la innovación.

Por exigencias de los proyectos de investigación que se desarrollan en las facultades de la universidad que demanda de los profesores universitarios profundizar en temas pedagógicos para dar salidas a las diferentes acciones del proyecto al que tributan en las áreas donde realiza el trabajo científico metodológico como vía certera mediante la cual se perfecciona el proceso de enseñanza -aprendizaje en el postgrado. Es necesario además contribuir por esta vía al perfeccionamiento de la Educación Superior mediante la formación permanente del profesor universitario con acciones de salida en las actividades sustantivas formación, investigación y extensión universitaria.

\section{Conclusiones}

A través de la literatura revisada, se valora la concepción de la sistematización como método, que tributa a la transformación de la práctica desde la teoría, posibilitando elevar la calidad e integración de los elementos aislados que hoy existen en la formación pedagógica del profesor universitario al constituirse en el enfoque sistémico de todas y cada una de las epatas y acciones que la concepción de la sistematización de la formación pedagógica postgraduada adopte. Desde esta lógica se da la intencionalidad de la sistematización, pues potencia el perfeccionamiento de la formación postgraduada con mayor protagonismo del profesor universitario.

Un punto coincidente ha sido el de destacar la importancia de determinar el objetivo, el objeto y el eje de sistematización, donde el taller como espacio de reflexión para perfeccionar la formación pedagógica del profesor universitario, posibilita la problematización (comprensión y análisis de la práctica por los que participan en la sistematización), la teorización en las modalidades de formación pedagógica sistematizadas (cómo debe ser, confrontación de conocimientos, modificación de conocimientos teóricos existentes en relación con las prácticas docentes) y los resultados de la sistematización(reconstrucción y ordenamiento de la práctica, socialización de los aprendizajes).

\section{Referencias bibliográficas}

Baute Álvarez, L.M. e Iglesias León, M. (2010). La formación del profesor universitario: experiencia de una sistematización. Evento Internacional Paulo Freire in Memoria. Cienfuegos.

Freire, Paulo. (2019). La importancia de leer y el proceso de liberación. Editorial Siglo XXI, S.A. de C.V., México, D.F.

Freire, Paulo. (2015). Pedagogía de la Autonomía. Editorial Siglo XXI, S.A. de C.V., México, D.F 


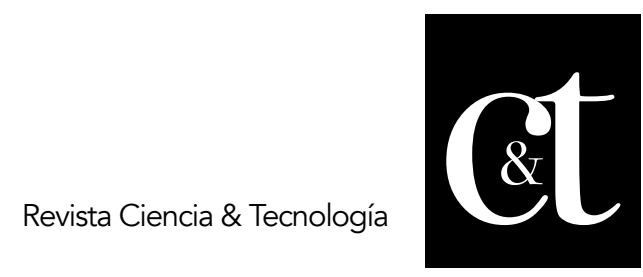

No. 27, 31 de julio de 2020

ISSN impreso: 1390 - 6321

ISSN online: 2661 - 6734

Freire, P. (2010). Pedagogía de la Autonomía y otros textos. La Habana: Editorial Caminos.

Fuentealba Jara, R., \& Imbarack Dagach, P. (2014). Compromiso docente, una interpelación al sentido de la profesionalidad en tiempos de cambio. Estudios pedagógicos (Valdivia), 40 (ESPECIAL), 257-273.

García Batista, Gilberto y Addine Fernández, Fátima. (n.d.) Currículo y profesionalidad del docente. MINED. La Habana. Editorial Pueblo y Educación.

González Maura, V. (1999). El profesor universitario, ¿un facilitador o un orientador en la educación en valores? Revista Cubana de Educación Superior. Volumen XIX (3): pp.12-14.

Jara Holliday, Óscar. (2008). Sistematización de Experiencias: un concepto enraizado en la realidad latinoamericana. Centro de Estudios y publicaciones Alforga, Costa Rica. Revista Internacional Digital Magisterio. Educación y Pedagogía. No 33 (3): pp. 15-19 Bogotá, Colombia.

Lucio-Villegas, E. (2015). Paulo Freire: La educación como instrumento para la justicia social. Revista Internacional de Educación para la Justicia Social, 4(1), 920.

Mejía, M. R. (2014). La educación popular: una construcción colectiva desde el sur y desde abajo. Education Policy Analysis Archives/Archivos Analíticos de Políticas Educativas, 22, 1-31. 